\title{
An investigation of thermo-fluid dynamic performance of a Stirling engine regenerator by means of OpenFOAM
}

\author{
Maria Faruoli ${ }^{1,2^{*}}$, Annarita Viggiano ${ }^{1}$, Vinicio Magi ${ }^{1}$ \\ ${ }^{1}$ School of Engineering, University of Basilicata, Viale dell'Ateneo Lucano 10, Potenza 85100, Italy \\ ${ }^{2}$ Environmental and Applied Fluid Dynamics Department, von Karman Institute for Fluid Dynamics, Sint-Genesius-Rode, B- \\ 1640, Belgium
}

Corresponding Author Email: maria.faruoli@unibas.it

https://doi.org/10.18280/mmc_b.870306

Received: 6 April 2018

Accepted: 19 May 2018

\section{Keywords:}

Stirling engine, regenerator, OpenFOAM, computational fluid dynamics

\begin{abstract}
A good design of the regenerator of a Stirling engine is required to obtain high performance and efficiency of such an engine. The regenerator is basically a heat-exchanger placed between the hot and cold working streams. It usually consists of stacked woven wires. The fluid pressure drop and heat transfer are the main parameters of the regenerator influencing the engine performance. In this work, friction coefficient, thermal efficiency and Nusselt number are numerically evaluated in order to assess the performance of the regenerator.

The open-source software OpenFOAM is used to analyse the thermo-fluid dynamic behaviour of a regenerator wire netting at different Reynolds numbers. Firstly, isothermal air flows and adiabatic wire matrices are considered, by assuming the fluid flowing through the regenerator as incompressible. Then, air flows with a fluid temperature of $500 \mathrm{~K}$ and wires at a temperature of $300 \mathrm{~K}$ are analysed. The results are compared with those obtained by means of the commercial software Ansys Fluent.
\end{abstract}

\section{INTRODUCTION}

In the last two centuries, the Stirling engine has been considered an interesting alternative to the classical steam engine or to the internal combustion engine. Indeed, the Stirling engine is characterized by high reliability and safety, even if the specific power is lower than the other two engines. The Stirling engine is an external combustion engine and various kinds of heat sources can be used to feed it. This engine uses compressible fluids as working fluids, so that air is often used.

The Stirling engine applications range from heating/cooling systems to mechanical propulsion and electric generation systems.

The design of a Stirling engine is mostly related to two main aspects:

(1) thermodynamic design, consisting of, for instance, the sizing of the heat exchanger and the regenerator;

(2) context design, consisting of combining the choice of the operating conditions (e.g. the working fluid) and the proposed application.

Specifically, this work focuses on the thermodynamic design of the regenerator, since its efficiency is proved to be directly related to the efficiency of the engine [1].

The regenerator is a heat exchanger, placed between the hot and cold streams. The purpose is to store the thermal heat taken from the hot stream and to release it to the cold stream, in order to increase the thermal efficiency of the engine [2]. Different types of regenerator are available. Among the others, the most used is the stoked woven wire regenerator type. It consists of metallic wires organized in ordered structures, generally kept at a fixed temperature. Generally, the performance of this engine component is given in terms of friction coefficient, thermal efficiency and Nusselt number, in order to assess both pressure losses and efficiency. Indeed, an increase of the pressure loss leads to a decrease of the engine power. The thermal performance of the regenerator is studied by analysing the interaction between the gas flow and the wires surfaces. Computational Fluid Dynamics (CFD) is a suitable tool to study the thermo-physical phenomena of the fluid flow in heat exchangers of different types [3-4]. As far as stocked woven wire regenerators are concerned, several studies have been performed by means of CFD. Costa et al. [3] analysed the pressure drop in a wire stacked regenerator type and its thermal efficiency in terms of Nusselt number [5]. Then, in Ref. [6], they carried out a study of the same configuration as porous media. In [7-9] the complete engine working process is simulated together with the regenerator performance and both friction coefficient and thermal heat have been evaluated. In all these works, Ansys Fluent has been employed for such simulations.

Several other studies have been carried out by using different numerical solvers to assess the performance of the regenerator, as shown in $[2,10]$.

The aim of this work is to perform accurate simulations of the thermo-fluid dynamic behaviour of the regenerator by means of an open source computational library, named OpenFOAM. To the best of authors' knowledge, this is the first time that such a software is used to compute the efficiency of a stoked woven wire regenerator. The results of the simulations are validated against computational and experimental data available in the literature.

This work is organized as follows: first the mathematical model is described, along with some useful definitions, then 
the computational setup is given, the results are discussed and, finally, the conclusions are summarized.

\section{THE MODEL}

The numerical modeling of the flow past a regenerator can be performed by solving the steady Reynolds-Averaged Navier-Stokes (RANS) equations for the mass, momentum and energy:

$$
\nabla \cdot(\rho \mathbf{u})=0
$$

$$
\nabla \cdot(\rho \mathbf{u u})-\nabla \cdot\left[\left(\mu+\mu_{t}\right) \nabla \mathbf{u}\right]+\nabla p=0
$$

$\nabla \cdot[\mathbf{u}(\rho E+p)]-\nabla \cdot\left[\left(\lambda+\lambda_{t}\right) \nabla T\right]=0$

where: $\rho$ is the gas density; $\boldsymbol{u}$ is the gas velocity; $\mu$ and $\mu_{\mathrm{t}}$ are the molecular and turbulent dynamic viscosity, respectively; $p$ is the pressure; $E$ is the total energy; $\lambda$ and $\lambda_{t}$ are the gas thermal conductivity and turbulent thermal conductivity, respectively; $T$ is the gas temperature. In the energy equation, the viscous dissipation term is not included. Indeed, the Brinkman number, $\mathrm{Br}$, is lower than unity, thus the viscous heating can be neglected.

The use of commercial software for the study of the regenerator performance is widespread. However, several open source codes are also available. Among the others, OpenFOAM (Open Source Field Operation and Manipulation) is a $\mathrm{C}++$ library, used primarily to create executables, known as applications [11]. The applications can be divided into solvers, which are designed to solve a specific problem in continuum mechanics, and utilities, which are created to perform tasks that involve data manipulation. Besides the precompiled applications, the user can create its own or modify the existing ones, introducing new equations or boundary conditions. The opportunity to modify the source codes and introduce new solvers makes the choice of OpenFOAM very attractive.

OpenFOAM has been assessed to be a valid alternative to commercial software for different applications. The aim of this work is to show the applicability of this software to model the Stirling engine regenerator in order to predict the regenerator performance under different operating conditions. The results are compared with those obtained by using Ansys Fluent and with measurements available in the literature.

Two different flow conditions are studied: firstly, the fluid is considered as incompressible and thermal phenomena are neglected, i.e. wires are adiabatic and the fluid is set at a constant temperature in the entire domain; then, the fluid is considered as compressible and the heat transfer between flow and wires is taken into account. Both laminar and turbulent cases are analysed. The definition of laminar and turbulent flows is not directly related to the Reynolds number as in a pipe but a transition between the two regimes is observed at Re equal to 500. Above this value, the flow is considered as fully turbulent and the numerical modeling of the turbulence is necessary to accurately reproduce the phenomena in the regenerator.

Moreover, the Reynolds number is kept relatively low (the maximum value considered is $\mathrm{Re}=1200$ ) in order to avoid large Mach numbers in the flow, that would lead to a reduction of the engine performance. Specifically, the ratio $\mathrm{Ma} / \mathrm{Re}$ is used as a discriminant value. This ratio is shown to be independent on the velocity, but depends on pressure, hydraulic diameter and flow properties [12]. In this work, this ratio is about $1.33 \cdot 10^{-4}$ based on the working pressure (atmospheric) and on the hydraulic diameter. Therefore, to keep the inlet value of Ma lower than 0.2, a maximum value of 1500 for $\mathrm{Re}$ is required.

Steady flow conditions are considered and a comparison with available results of other authors is presented. In the case of oscillating flows, the friction coefficients are estimated to be $30 \%$ higher than the theoretical value of one-directional flows [13].

Firstly, the fluid is considered as incompressible and walls are adiabatic and the results are compared with measurements of Tanaka et al. [14]. The incompressibility assumption is acceptable until heat transfer is not included. Indeed, according to Organ [12], from the compressibility vulnerability chart for air, the incompressibility assumption is acceptable only for low $\operatorname{Re}$ numbers $(\operatorname{Re}<100)$ if thermal variations in the flow are considered. Thus, for the nonisothermal cases, the compressibility effect will not be neglected.

The regenerator is generally composed by matrices of wires netting stacked next, or on top, of each other. The configuration considered is a misaligned wires distribution that is also employed by Bello et al. [13]. The results of [13] are obtained by means of Ansys Fluent and are used as a reference. In this work, computations based only on OpenFOAM are performed.

The computational domain is a parallelepiped with a crosssection of $1 \mathrm{~mm} \times 1 \mathrm{~mm}$ and a streamwise length of $5 \mathrm{~mm}$. The wire matrix is placed $2 \mathrm{~mm}$ downstream the inlet section, and its width, $L$, is $0.922 \mathrm{~mm}$.

The wire matrix is characterized by the porosity, $\pi_{v}$ and the hydraulic diameter, $d_{h}$. The porosity is given by:

$\pi_{v}=\frac{V_{t o t}-V_{m}}{V_{t o t}}$

where $V_{t o t}$ is the total volume of the matrix and $V_{m}$ is the volume occupied by the wires.

Based on the matrix porosity, the hydraulic diameter is:

$d_{h}=\frac{4 \pi_{v}}{\phi\left(1-\pi_{v}\right)}$

where $\phi$ is the ratio between the surface area and the volume of the woven matrix. For the geometry under investigation, the parameters used for the computations are reported in Table 1.

Table 1. Geometric parameters of the regenerator matrix

\begin{tabular}{cccc}
\hline $\mathbf{d}_{\mathbf{w}}[\mathbf{m m}]$ & $\boldsymbol{\pi}_{\mathbf{v}}[-]$ & $\mathbf{d}_{\mathbf{h}}[\mathbf{m m}]$ & $\mathbf{L}[\mathbf{m m}]$ \\
\hline 0.11 & 0.641 & 0.1728 & 0.922 \\
\hline
\end{tabular}

The Reynolds number is given by:

$\operatorname{Re}=\frac{\rho u_{\max } d_{h}}{\mu}$

where $u_{\max }=u_{i n} / \pi_{v}$ with $u_{\text {in }}$ the inlet velocity. 
The results of the simulations are given in terms of friction coefficient and thermal efficiency. These dimensionless parameters are given in the following:

$c_{f}=\frac{\Delta p}{\left(\frac{\rho}{2} \frac{L}{d_{h}} u_{\max }^{2}\right)}$,

$\eta=\frac{\dot{Q}}{\dot{Q}_{i d}}$

In Eq. (7) and Eq. (8), $\Delta p=p_{\text {in }}-p_{\text {out }} ; \dot{Q}$ and $\dot{Q}_{i d}$ are the total effective and the ideal thermal power, respectively, transferred between walls and fluid. The ideal transferred thermal power is computed as:

$\dot{Q}_{i d}=\dot{m}\left[c_{p}\left(T_{i n}-T_{w a l l}\right)+\frac{u_{i n}{ }^{2}}{2}\right]$

where $\dot{m}$ represents the mass flow rate; $c_{p}$ is the gas specific heat at constant pressure; $T_{i n}$ is the gas temperature at the inlet section; $T_{\text {wall }}$ is the temperature of the wires.

As regards the regenerator efficiency, the Nusselt number is evaluated as:

$N u=\frac{h_{e x} d_{h}}{\lambda}$,

where $\lambda$ is the gas thermal conductivity and $h_{e x}$ represents the heat exchange coefficient, and it is computed as:

$h_{e x}=\frac{\dot{Q}}{A\left(T_{\text {in }}-T_{\text {out }}\right)}$,

where $\mathrm{A}$ is the matrix walls area and $T_{\text {out }}$ is the outlet gas temperature.

\section{NUMERICAL SETUP}

Figure 1 shows a sketch of the computational domain with boundary specifications.

The same numerical grid employed by Bello et al. [13] has been used and consists of approximately 1.5 million tetrahedral elements. A blow-up of the grid near the netting wires is shown in Figure 2. In order to guarantee accuracy, a correction in the solver set up for the non-orthogonality of the mesh (nNonOrthogonalCorrectors 2 in fvSolution file) is included.

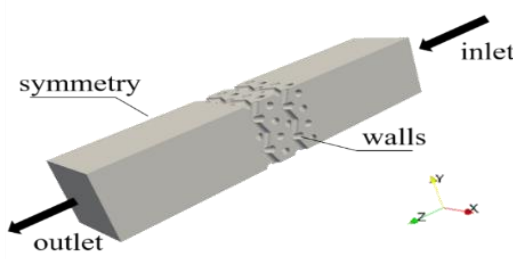

Figure 1. Computational domain and definition of the boundaries

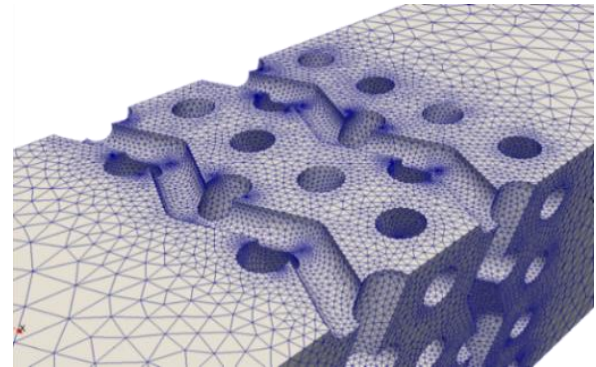

Figure 2. Blow-up of the computational grid near the netting wires

\subsection{Incompressible case}

In the case of incompressible fluid with no heat transfer, the flow is described by the governing equations for mass (Eq. (1)) and momentum (Eq. (2)).

Among the solvers of the OpenFOAM 4.0 release, the simpleFoam solver has been chosen. It is a steady-state solver for isothermal, incompressible and turbulent flows, based on a SIMPLE algorithm.

The flow properties are set as constant. Specifically, in Fluent the density and the dynamic viscosity are fixed, while in OpenFOAM only the kinematic viscosity is set in the transportProperties file. The values used are reported in Table 2 .

Table 2. Fluid properties for incompressible cases

\begin{tabular}{ccc}
\hline Density & Dynamic Viscosity & Kinematic viscosity \\
\hline $1.225 \mathrm{~kg} \mathrm{~m}^{-3}$ & $1.7894 \mathrm{e}-05 \mathrm{~kg} \mathrm{~m}^{-1} \mathrm{~s}^{-1}$ & $1.4607 \mathrm{e}-05 \mathrm{~m}^{2} \mathrm{~s}^{-1}$ \\
\hline
\end{tabular}

Besides mass and momentum conservations, a turbulence model is used for the closure problem of the Navier-Stokes system. In this work, the RNG $k-\varepsilon$ model is applied based on its accuracy near the walls of the Stirling regenerator [3]. The equations solved for the turbulent kinetic energy, $k$, and the turbulent dissipation rate, $\varepsilon$, are:

$\nabla \cdot(\rho k \mathbf{u})=\nabla \cdot\left[\left(\mu+\frac{\mu_{t}}{\sigma_{k}}\right) \nabla k\right]+P_{k}-\rho \varepsilon$

$\nabla \cdot(\rho \epsilon \mathbf{u})=\nabla \cdot\left[\left(\mu+\frac{\mu_{t}}{\sigma_{\epsilon}}\right) \nabla \epsilon\right]+\frac{C_{1_{\epsilon}} \epsilon}{k} P_{k}-\frac{C_{2 \epsilon}^{*} \rho \epsilon^{2}}{k}$.

In Eq. (12) $\sigma_{k}$ is a model parameter and $\mathrm{P}_{\mathrm{k}}$ is the production term. In Eq. (13) $\sigma_{\epsilon}, \mathrm{C}_{1 \epsilon}$ and $C_{2 \epsilon}{ }^{*}$ are model parameters.

Both software is based on a Finite Volume Method (FVM). In Fluent, mass and momentum equations are solved by using a second-order upwind scheme, whereas a first-order upwind scheme is used for the $k$ and $\varepsilon$ equations. In OpenFOAM, the same scheme is used for the turbulent variables, while for the mass and the momentum equation the so-called linearUpwind scheme is chosen. It corresponds to a second order, upwindbiased, unbounded scheme, and a specified discretization of the velocity gradient is needed.

\subsection{Compressible case}

In the case of compressible flow, in addition to mass and momentum equations, a transport equation for the energy is solved, i.e. Eq. (3). 
Among the available solvers of OpenFOAM 4.0, the rhoSimpleFoam solver is chosen. It is a steady-state solver of RANS equations for laminar and turbulent flows of compressible fluids, based on the SIMPLE algorithm. The use of this solver, together with the choice of the enthalpy as energy variable in the thermophysicalProperties file, guarantees that the same equations implemented in Ansys Fluent are used.

The fluid considered in the regenerator is air, characterized by the following properties:

(1) the molecular weight is $28.9 \mathrm{~kg} / \mathrm{kmol}$. The fluid is considered as bimolecular $(\gamma=1.4)$;

(2) the density is computed from the ideal equation of state;

(3) the dynamic viscosity is evaluated by the Sutherland's law:

$$
\mu=\mu_{\text {ref }}\left(\frac{T}{T_{r e f}}\right)^{\frac{3}{2}} \frac{T_{r e f}+S}{T+S},
$$

where $T_{r e f}$ is the reference temperature, $\mu_{r e f}$ is the reference viscosity and $S$ is the Sutherland temperature. These values are imposed equal to $273.15 \mathrm{~K}, 1.716 \mathrm{e}-5 \mathrm{kgm}^{-1} \mathrm{~s}^{-1}$ and $110.4 \mathrm{~K}$, respectively;

(1) the specific heat at constant pressure, $c_{p}$, is $1006.4 \mathrm{~J} / \mathrm{kgK}$. It is considered constant and independent on temperature;

(2) the thermal conductivity $\lambda$ is assumed constant and equal to $0.0242 \mathrm{~W} / \mathrm{mK}$. In order to keep such an assumption in both software, it was necessary to modify the implementation of the transport model in OpenFOAM. Indeed, among the existing models, the one that employs the Sutherland's law considers an empirical model for the thermal conductivity dependent on the temperature. The implementation has been modified, by generating a new transport model that allows to impose a constant value for $\lambda$ defined by the user.

As regards spatial discretization, the numerical schemes are the same as those used for the incompressible case for velocity, pressure and turbulent parameters. A second order upwind discretization for the energy equation in Fluent and a linearUpwind scheme in OpenFOAM is used.

\section{RESULTS}

\subsection{Incompressible Case}

Table 3. Boundary conditions at the inlet for different $\mathrm{Re}$

\begin{tabular}{cccc}
\hline $\mathbf{R e}$ & $\mathbf{U}\left[\mathbf{m ~ s}^{\mathbf{- 1}}\right]$ & $\boldsymbol{k}\left[\mathbf{m}^{\mathbf{2}} \mathbf{s}^{-\mathbf{2}}\right]$ & $\boldsymbol{\varepsilon}\left[\mathbf{m}^{\mathbf{2}} \mathbf{s}^{\mathbf{3}}\right]$ \\
\hline 5.35 & 0.29 & 1 & 100 \\
\hline 11.37 & 0.62 & 1 & 100 \\
\hline 20.18 & 1.09 & 10 & 1000 \\
\hline 92.35 & 5.00 & 10 & 1000 \\
\hline 196.65 & 10.65 & 10 & $10^{4}$ \\
\hline 314.06 & 17.01 & 100 & $10^{5}$ \\
\hline 614.41 & 33.27 & 100 & $10^{6}$ \\
\hline $1,023.55$ & 55.43 & 1000 & $10^{6}$ \\
\hline
\end{tabular}

In the case of incompressible fluid, the boundary conditions are chosen in order to get a Reynolds number that ranges approximately from 5 to 1000 . A uniform velocity profile at the inlet has been set. A fixed value for the pressure, i.e. atmospheric pressure, has been set at the outlet boundary. The wires are considered as stationary walls and adiabatic. The initial values for the turbulent kinetic energy $(k)$ and the turbulent dissipation rate $(\epsilon)$ in the domain are $10^{-5} \mathrm{~m}^{2} \mathrm{~s}^{-2}$ and $10^{-7} \mathrm{~m}^{2} \mathrm{~s}^{-3}$ respectively for all the simulations. To guarantee numerical stability, values at the inlet closer to the final solution in the domain are needed. Table 3 summarizes for each simulation the inlet boundary conditions for velocity and $k$ and $\varepsilon$ values, chosen in order to achieve a good convergence.

In Figure 3 the friction coefficient is shown as a function of the Reynolds number. The results are compared with those provided by Bello et al. [13], and the empirical relation provided by Tanaka et al. [14]. The figure shows a good agreement between the results of Fluent and OpenFOAM, while a discrepancy with the experimental results was expected because of the assumption of steady flow.

In Figure 4 the average pressure drop along the streamwise direction is shown for $\mathrm{Re}=92$. The pressure profile shows a series of steps due to the wires in the fluid path that influence the flow leading to a local reduction of the pressure. The profiles are overlapping on almost the entire domain, showing the capability of OpenFOAM.

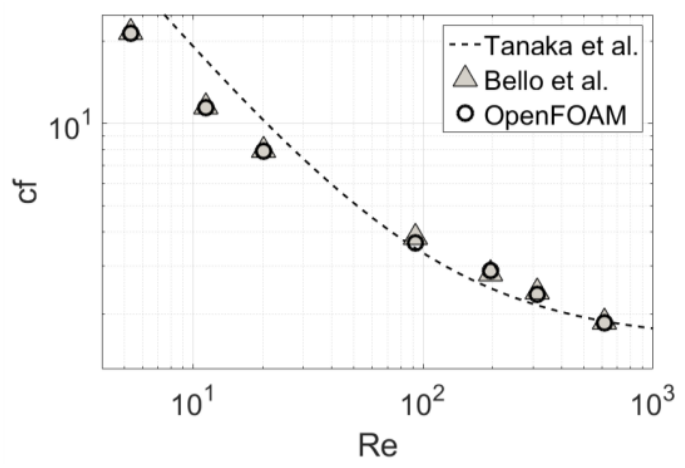

Figure 3. Friction coefficient versus Re for an isothermal flow

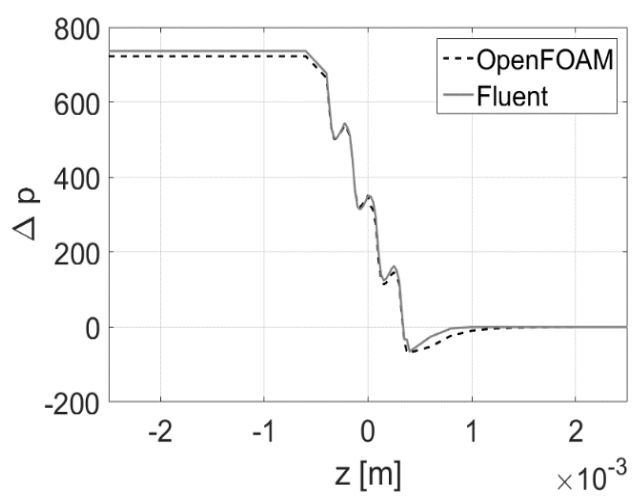

Figure 4. Pressure profile along $\mathrm{z}$-direction with isothermal flow $-\mathrm{Re}=92$

\subsection{Compressible case}

In this case, the wires are kept at a fixed temperature $\left(T_{\text {wall }}=300 \mathrm{~K}\right)$, whereas the inlet fluid temperature is $T_{\text {in }}=$ $500 K$. These values are set as boundary conditions for both software. Unlike the previous case, where the velocity was imposed on the inlet, the pressure is set at the inlet section as well as at the outlet section. This type of boundary condition is more suitable for the compressible flow and ensures better convergence and higher stability of the solver.

Boundary conditions for the static pressure drop, the turbulent kinetic energy and the turbulent dissipation rate are 
summarized in Table 4 for each Re.

Table 4. Boundary conditions at different Re for a compressible flow

\begin{tabular}{cccc}
\hline $\mathbf{R e}$ & $\boldsymbol{\Delta} \mathbf{p}[\mathbf{P a}]$ & $\mathbf{k}\left[\mathbf{m}^{\mathbf{2}} \mathbf{s}^{-\mathbf{2}}\right]$ & $\boldsymbol{\varepsilon}\left[\mathbf{m}^{\mathbf{2}} \mathbf{s}^{\mathbf{- 3}}\right]$ \\
\hline 7.84 & 39.5 & 5 & 1000 \\
\hline 24.61 & 195.6 & 10 & 1000 \\
\hline 185.60 & 5,236 & 100 & $10^{4}$ \\
\hline 659.70 & 53,000 & 500 & $7 \cdot 10^{6}$ \\
\hline 936.36 & 102,879 & 500 & $7 \cdot 10^{6}$ \\
\hline $1,278.96$ & 175,880 & 500 & $7 \cdot 10^{6}$ \\
\hline
\end{tabular}

In Figure 5, the friction coefficient is shown versus the Reynolds number. Specifically, the friction coefficient decreases up to about $\mathrm{Re}=500$ and then starts to rise when $\mathrm{Re}$ is higher than 600. Starting from $\mathrm{Re}=600$, compressibility effects are not negligible, leading to a modification in the flow behaviour. Indeed, even if the imposed pressure drop is almost twice, the velocity field changes are relatively small. The figure shows again a good comparison between the two solvers with a difference less than $3 \%$.

In Figure 6, the thermal efficiency as a function of Re is shown. For low Re, i.e. laminar flow, the thermal efficiency is close to unity. As $\mathrm{Re}$ increases, i.e. turbulent and compressibility become not negligible, the efficiency starts to decrease. This represents a further motivation to operate with low Re in such a regenerator. Again, the figure shows a good comparison between the two solvers with a difference less than $4 \%$.

Figure 7 shows the Nusselt number as a function of Re for the two solvers. The correlation between $\mathrm{Re}$ and $\mathrm{Nu}$ is quite similar for the two software. Indeed, the difference in the slope of the linear regression line is within $7 \%$.

Figure 8 and Figure 9 show the mean pressure drop and the mean temperature profiles, respectively, along the streamwise direction for $\mathrm{Re}=660$. As expected, the trend is quite similar to the incompressible case and, once more, a very good comparison between the two solvers is obtained, i.e. maximum difference less than $1 \%$. Figure 9 shows that the first row of wires is responsible for the highest decrease of temperature, due to the highest temperature gradient between the flow and the wire. Also for the temperature, the maximum difference between the two profiles is less than $1 \%$.

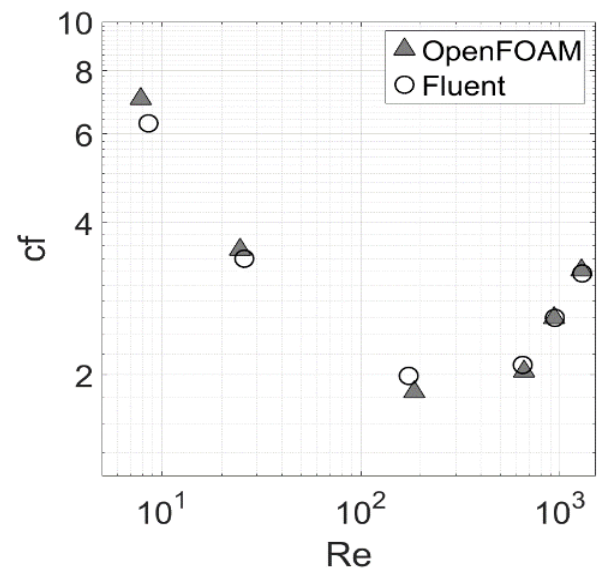

Figure 5. Friction coefficient as a function of Re for compressible flow, $\mathrm{T}_{\text {in }}=500 \mathrm{~K}$ and $\mathrm{T}_{\text {wall }}=300 \mathrm{~K}$

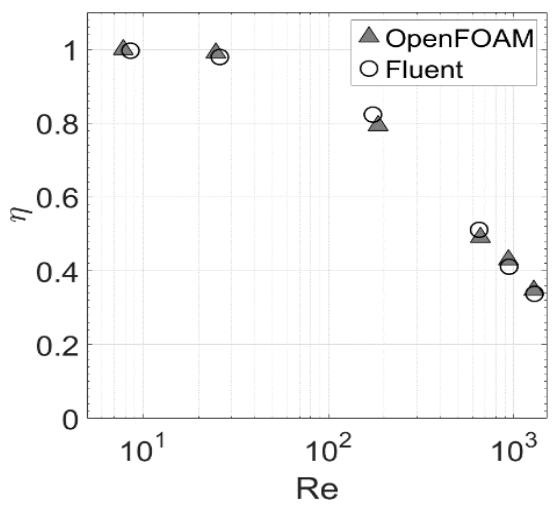

Figure 6. Thermal efficiency vs Re for compressible flow, $\mathrm{T}_{\text {in }}=500 \mathrm{~K}$ and $\mathrm{T}_{\text {wall }}=300 \mathrm{~K}$

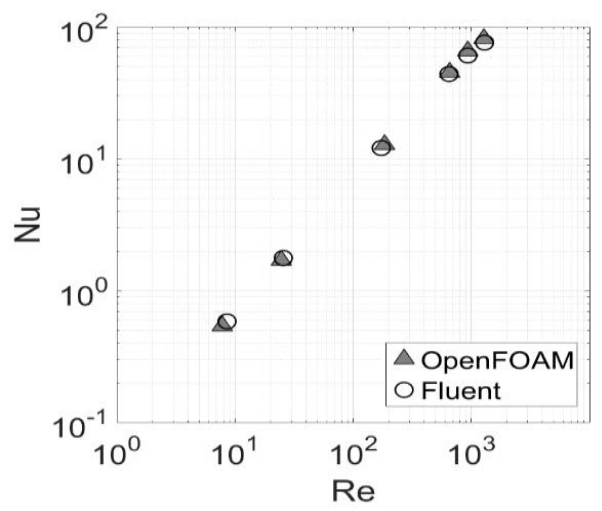

Figure 7. Nusselt number vs Re for compressible flow, $\mathrm{T}_{\text {in }}=500 \mathrm{~K}$ and $\mathrm{T}_{\text {wall }}=300 \mathrm{~K}$

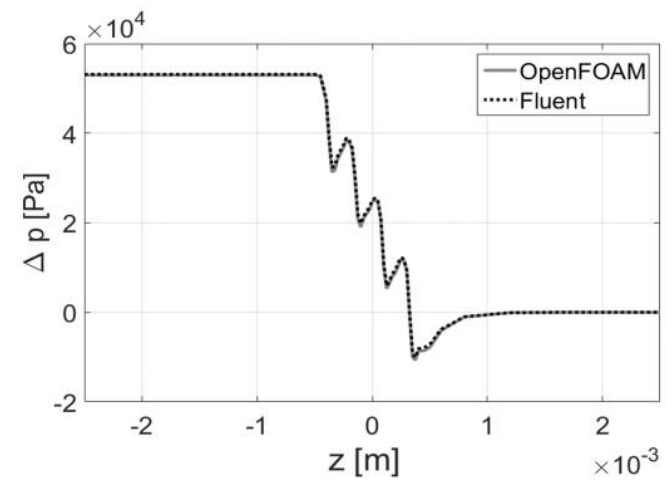

Figure 8. Pressure profile along z-direction for compressible flow, $\mathrm{T}_{\text {in }}=500 \mathrm{~K}$ and $\mathrm{T}_{\text {wall }}=300 \mathrm{~K}-\mathrm{Re}=660$

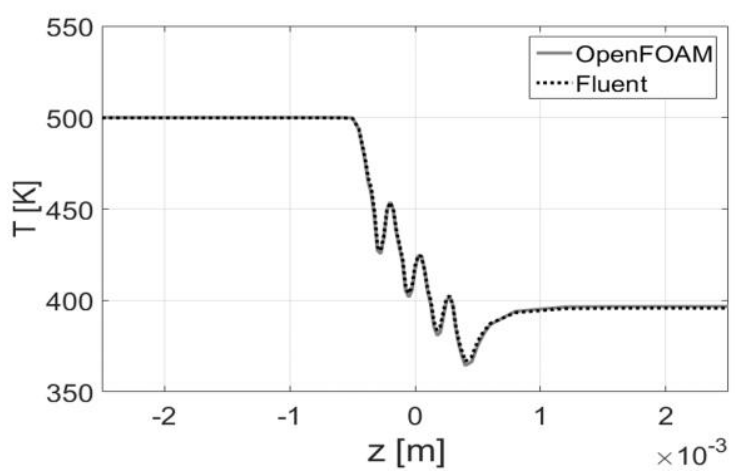

Figure 9. Temperature profile along z-direction for compressible flow, $\mathrm{T}_{\mathrm{in}}=500 \mathrm{~K}$ and $\mathrm{T}_{\text {wall }}=300 \mathrm{~K}-\mathrm{Re}=660$ 
In order to show the influence of the wires on the spatial distribution of flow properties, contour plots of different flow parameters are presented along the middle plane YZ. Mach number, gas density, pressure and temperature distributions are provided in Figure 10, Figure 11, Figure 12 and Figure 13, respectively. The figures refer to the case with $\mathrm{Re}=660$ and Mach number at the inlet section, $\mathrm{Ma}_{\mathrm{in}}$, equal to 0.14 . Figure 10 shows that the maximum value of $\mathrm{Ma}$ in the domain is about 0.55. For this Mach number, the effects of compressibility are not negligible and influence the flow characteristics. Indeed, Figure 11 shows that the density varies from a value around 0.9 to a value of about 1.43 . From the Mach number contour plots, it follows that OpenFOAM provides a somewhat higher velocity than Fluent, even if such a difference is not remarkable.

More evident are the differences for the gas density distribution. The trend is similar for both cases, but OpenFOAM predicts higher values than those of Fluent. Particularly, these differences are more evident close to the wires.

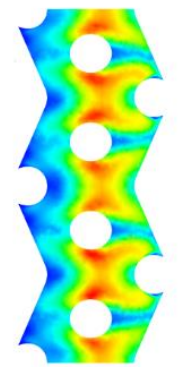

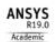

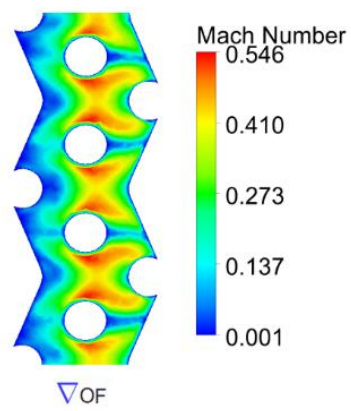

Figure 10. Mach number contour plots on YZ middle plane of the wires matrix in case of compressible flow, $T_{\text {in }}=500 \mathrm{~K}$ and $\mathrm{T}_{\text {wall }}=300 \mathrm{~K}-\mathrm{Re}=660$

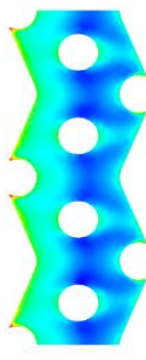

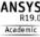

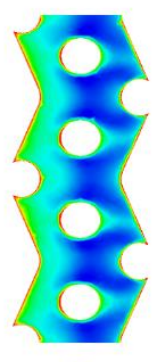

$\nabla$ OF

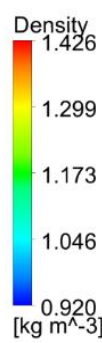

Figure 11. Density contour plots on $\mathrm{YZ}$ middle plane of the wires matrix in case of compressible flow, $T_{\text {in }}=500 \mathrm{~K}$ and

$$
\mathrm{T}_{\text {wall }}=300 \mathrm{~K}-\mathrm{Re}=660
$$

Figure 12 provides a good comparison of the two solvers for the pressure, as also shown in Figure 8. As regards Figure 13, similar temperature distributions for both solvers are given. However, OpenFOAM computes the gas temperatures slightly higher than Ansys. This discrepancy could be due to the difference between the numerical schemes of the two solvers. However, differences are not remarkable and the maximum difference is less than $5 \%$ in the entire flow field.

As an example, the temperature difference has been plotted at the outlet section as shown in Figure 14. In the figure, the results from the two software are interpolated by using the same technique, i.e. the temperature difference is computed at each grid point with the same interpolation procedure. The difference is shown to be within $2 \%$ for each grid point at the outlet section.
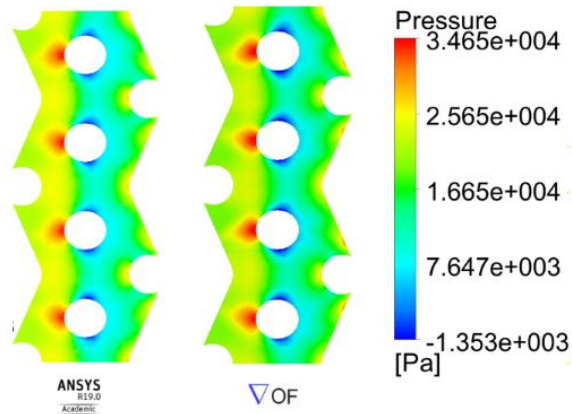

Figure 12. Relative pressure contour plots on $\mathrm{YZ}$ middle plane of the wires matrix in case of compressible flow, $\mathrm{T}_{\text {in }}=500 \mathrm{~K}$ and $\mathrm{T}_{\text {wall }}=300 \mathrm{~K}-\mathrm{Re}=660$
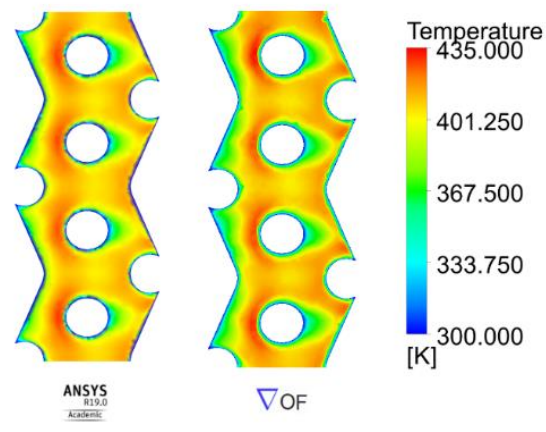

Figure 13. Temperature contour plots on $\mathrm{YZ}$ middle plane of the wires matrix in case of compressible flow, $\mathrm{T}_{\text {in }}=500 \mathrm{~K}$ and $\mathrm{T}_{\text {wall }}=300 \mathrm{~K}-\mathrm{Re}=660$
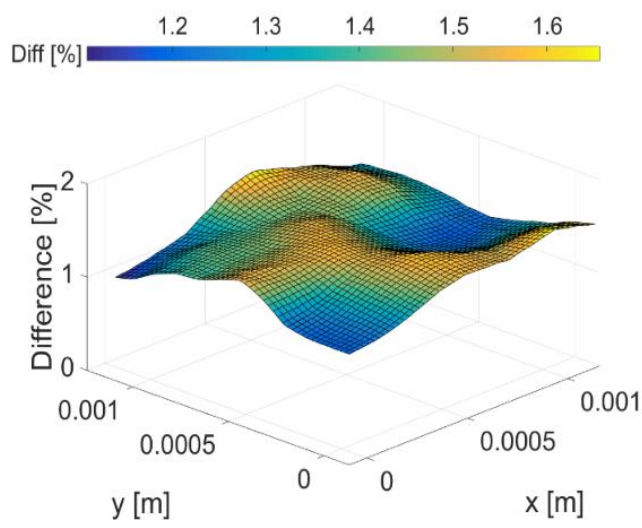

Figure 14. Percentage difference map for gas temperature at the outlet section for compressible flow, $\mathrm{T}_{\text {in }}=500 \mathrm{~K}$ and

$$
\mathrm{T}_{\text {wall }}=300 \mathrm{~K}-\mathrm{Re}=660
$$

\section{CONCLUSIONS}

In this work, a numerical study of a Stirling engine regenerator is presented. The regenerator is characterized by a misaligned matrix and it is modeled under steady state conditions by means of the OpenFOAM software. The main focus of this work is to assess the capability of this software to analyse the performance of the regenerator with different $\mathrm{Re}$ 
(from laminar to turbulent cases). Firstly, a simplified model is considered with no heat transfer and under incompressible flow assumption. Then, wire heat transfer has been included together with gas compressibility effects. The results are compared with those obtained by employing Ansys Fluent. A very good agreement is recovered, both in terms of dimensionless parameters and of velocity, temperature, pressure and density distributions. As regards dimensionless parameters, a maximum difference of the friction coefficient of about $3 \%$ is observed for both compressible and incompressible cases. Similarly, a good comparison is obtained for the thermal efficiency and the gas temperature. Indeed, as regards Nusselt number, the two software make a difference of about $7 \%$, whereas a maximum difference of the gas temperature less than $2 \%$ at the outlet section is recovered.

The results show that, for the incompressible case, the friction coefficient decreases by increasing Re. For the compressible case, the friction coefficient decreases by increasing $\operatorname{Re}$ when $\mathrm{Re}$ is less than 170 , whereas for higher values of $\mathrm{Re}$ an opposite trend is obtained. The thermal efficiency/Nusselt number decreases/increases when $\mathrm{Re}$ increases. On the basis of these results, it can be concluded that OpenFOAM is suitable to analyse the performance of the Stirling regenerator and provides an effective numerical tool for additional analysis of such an engine.

\section{ACKNOWLEDGMENT}

The von Karman Institute for Fluid Dynamics is acknowledged for the availability of computing resources.

\section{REFERENCES}

[1] Martini WR. (1978). Stirling engine design manual, US Department of Energy, Office of Conservation and Solar Applications, Division of Transportation Energy Conservation. DOE/NASA/3194-1.

[2] Andersen SK, Carlsen H, Thomsen PG. (2006). Numerical study on optimal Stirling engine regenerator matrix designs taking into account the effects of matrix temperature oscillations. Energy Convers. Manag. 47(78): https://doi.org/10.1016/j.enconman.2005.06.006 $894-908$.

[3] Costa SC, Barrutia H, Esnaola JA, Tutar M. (2013). Numerical study of the pressure drop phenomena in wound woven wire matrix of a Stirling regenerator. Energy Convers. Manag. 67: 57-65. https://doi.org/10.1016/j.enconman.2012.10.014

[4] Asif M, Aftab H, Syed HA, Ali MA, Muizz PM. (2017). Simulation of corrugated plate heat exchanger for heat and flow analysis. International Journal of Heat and Technology 35(1): 205-210. https://doi.org/10.18280/ijht.350127

[5] Costa SC, Barrutia H, Esnaola JA, Tutar M. (2014). Numerical study of the heat transfer in wound woven wire matrix of a Stirling regenerator. Energy Convers. Manag 79: 255-264. https://doi.org/10.1016/j.enconman.2013.11.055

[6] Costa SC, Barreno I, Tutar M, Esnaola JA, Barrutia H. (2015). The thermal non-equilibrium porous media modelling for CFD study of woven wire matrix of a Stirling regenerator. Energy Convers. Manag 89: 473-

483. https://doi.org/10.1016/j.enconman.2014.10.019

[7] Xiao G, et al. (2017). Design optimization with computational fluid dynamic analysis of $\beta$-type Stirling engine, Appl. Therm. Eng. 113: 87-102. https://doi.org/10.1016/j.applthermaleng.2016.10.063

[8] Li Z, Haramura Y, Kato Y, Tang D. (2014). Analysis of a high performance model Stirling engine with compact porous-sheets heat exchangers. Energy 64: 31-43. https://doi.org/10.1016/j.energy.2013.11.041

[9] Abuelyamen A, Ben-Mansour R, Abualhamayel H, Mokheimer EMA. (2017). Parametric study on beta-type Stirling engine. Energy Convers. Manag. 145: 53-63. https://doi.org/10.1016/j.enconman.2017.04.098

[10] Mahkamov K. (2006). Design improvements to a biomass Stirling engine using mathematical analysis and 3D CFD modeling. J. Energy Resour. Technol. 128(3): 203. https://doi.org/10.1115/1.2213273

[11] Greenshields C. (2018). OpenFOAM | The OpenFOAM Foundation, OpenFOAM. https://openfoam.org/, accessed: 23-Mar-2018.

[12] Organ AJ. (2007). The air engine: Stirling cycle power for a sustainable future. Boca Raton: CRC Press.

[13] Bello F, Viggiano A, Fanelli E, Magi V. (2014). A CFD analysis of the air flow through the matrix regenerator of Stirling engines. in ISEC Conference Proceedings, pp. 58-71.

[14] Tanaka M, Yamashita I, Chisaka F. (1990). Flow and heat transfer characteristics of the Stirling engine regenerator in an oscillating flow. JSME Int. J. Ser 2 Fluids Eng. Heat Transf. Power Combust. Thermophys. Prop. 33(2): 283-289. https://doi.org/10.1299/jsmeb1988.33.2_283

\section{NOMENCLATURE}

A

$\mathrm{Br}$

$\mathrm{c}_{\mathrm{p}}$

$\mathrm{d}_{\mathrm{h}}$

$h_{\text {ex }}$

E

$\mathrm{k}$

$\mathrm{L}$

$\mathrm{Ma}$

$\mathrm{Nu}$

$\mathrm{p}$

$\dot{Q}$

$\dot{Q}_{i d}$

$\operatorname{Re}$

$\mathrm{S}$

$\mathrm{T}$

$\mathrm{T}_{\text {wall }}$

$\mathrm{u}$

$\mathrm{u}_{\max }$

$\mathrm{V}_{\mathrm{m}}$

$\mathrm{V}_{\text {tot }}$

\section{Greek symbols}

Y

$\varepsilon$

$\eta$
Matrix walls area, $\mathrm{m}^{2}$

Brinkman number

Specific heat at constant pressure, $\mathrm{J} . \mathrm{kg}^{-1}$. $\mathrm{K}^{-1}$

Hydraulic diameter, $m$

Heat exchange coefficient

Total energy, $\mathrm{m}^{2} \cdot \mathrm{s}^{-2}$

Turbulent kinetic energy, $\mathrm{m}^{2} \cdot \mathrm{s}^{-2}$

Matrix axial length, $\mathrm{m}$

Mach number

Nusselt number

Pressure, $\mathrm{Pa}$

Exchanged thermal power, $\mathrm{W}$

Ideal exchanged thermal power, $\mathrm{W}$

Reynolds number

Sutherland temperature, K

Temperature, $\mathrm{K}$

Wires temperature, $\mathrm{K}$

Velocity, $\mathrm{m} . \mathrm{s}^{-1}$

Ratio between the frontal maximum velocity and the porositv. $\mathrm{m} . \mathrm{s}^{-1}$

Wires total volume, $\mathrm{m}^{3}$

Matrix total volume, $\mathrm{m}^{3}$
Specific heat ratio

Turbulent dissipation rate, $\mathrm{m}^{2}-\mathrm{s}^{-3}$

Thermal efficiency 
Dynamic viscosity, kg. $\mathrm{m}^{-1} \cdot \mathrm{s}^{-1}$

Matrix volumetric porosity

Density, kg.m ${ }^{-3}$

\section{Subscripts}

in

out

ref

$\mathrm{t}$
Inlet section

Outlet section

Reference value

Turbulent properties 